\title{
BASI74, a Virulence-Related sRNA in Brucella abortus
}

\author{
Hao Dong ${ }^{1 t}$, Xiaowei Peng ${ }^{2 t}$, Yufu Liu ${ }^{2,3 t}$, Tonglei Wu ${ }^{4}$, Xiaolei Wang ${ }^{5}$, Yanyan $\mathrm{De}^{6}$, \\ Tao Han', Lin Yuan', Jiabo Ding ${ }^{2}$, Chuanbin Wang ${ }^{1 *}$ and Qingmin Wu ${ }^{6 *}$ \\ ${ }^{1}$ China Animal Disease Control Center, Beijing, China, ${ }^{2}$ Department of Inspection Technology Research, China Institute \\ of Veterinary Drug Control, Beijing, China, ${ }^{3}$ College of Veterinary Medicine, South China Agricultural University, Guangzhou, \\ China, ${ }^{4}$ Key Laboratory of Preventive Veterinary Medicine of Hebei Province, Hebei Normal University of Science \\ and Technology, Qinhuangdao, China, ${ }^{5}$ Institute of Animal Husbandry and Veterinary Medicine, Beijing Academy \\ of Agriculture and Forestry Sciences, Beijing, China, ${ }^{6}$ Key Laboratory of Animal Epidemiology and Zoonosis, Ministry \\ of Agriculture, College of Veterinary Medicine, China Agricultural University, Beijing, China
}

Brucella spp. are intracellular pathogens that infect a wide variety of mammals including humans, posing threats to the livestock industry and human health in developing countries. A number of genes associated with the intracellular trafficking and multiplication have so far been identified in Brucella spp. However, the sophisticated post-transcriptional regulation and coordination of gene expression that enable Brucella spp. to adapt to changes in environment and to evade host cell defenses are not fully understood. Bacteria small RNAs (sRNAs) play a significant role in post-transcriptional regulation, which has already been confirmed in a number of bacteria but the role of sRNAs in Brucella remains elusive. In this study, we identified several different sRNAs in Brucella spp., and found that over-expression of a sRNA, tentatively termed BASI74, led to alternation in virulence of Brucella in macrophage infection model. The expression level of BASI74 increased while Brucella abortus 2308 was grown in acidic media. In addition, BASI74 affected the growth ratio of the Brucella cells in minimal media and iron limiting medium. Using a two-plasmid reporter system, we identified four genes as the target of BASI74. One target gene, BABl1154, was predicted to encode a cytosineN4-specific DNA methyltransferase, which protects cellular DNA from the restriction endonuclease in Brucella. These results show that BASI74 plays an important role in Brucella survival in macrophage infection model, speculatively by its connection with stress response or impact on restriction-modification system. Our study promotes the understanding of Brucella sRNAs, as well as the mechanism by which sRNAs use to influence Brucella physiology and pathogenesis.

Keywords: Brucella, sRNA, virulence, post-transcriptional regulation, stress response, intracellular survival

\section{INTRODUCTION}

Brucella spp. as well as other bacteria are capable of quickly adapting to changing conditions to survive. Successful adaptation depends on changes in gene expression, which may take place at both transcriptional level and post-transcriptional level. Compared to a wide range of studies in transcriptional regulation, e.g., transcriptional regulators (Dong et al., 2013), two-component regulators (Abdou et al., 2013), quorum sensing systems (Brambila-Tapia and Perez-Rueda, 2014), 
only a limited number of studies focused on post-transcriptional regulation [especially small RNAs (sRNAs)] in Brucella spp.

Small RNAs usually have a length of 50-300 nt and most of them base-pair with mRNA and regulate mRNA stability or mRNA translation efficiency. According to the location of their genes on the chromosomes, sRNAs can be divided into two groups: (a) cis-acting sRNAs with the capacity of extensive base pairing, and (b) trans-encoded sRNAs, having limited potential of base pairing with the target mRNAs (Waters and Storz, 2009).

Previous studies have demonstrated that some sRNAs are involved in bacterial virulence in various pathogens (such as Listeria, Salmonella, Vibrio, and Yersinia). Two sRNAs (AbcR1 and AbcR2) regulating Brucella virulence were identified, and AbcR1 and AbcR2 double mutant was defective in both macrophage infection model and mice chronic infection model (Caswell et al., 2012; Sheehan and Caswell, 2017). One sRNA (BSR0602), which modulated Brucella melitensis intracellular survival was also reported (Wang et al., 2015). Based on the results of strand-specific RNA deep-sequencing approach, 1321 sRNAs were found in $B$. melitensis $16 \mathrm{M}$, and one sRNA, BSR0441, involved in bacterial virulence in both macrophages and mice infection models was also found (Zhong et al., 2016).

In previous studies, we integrated the output of two published sRNA detection programs (sipht and napp), and found a total of 129 sRNAs candidates, out of which 7 from 20 sRNA candidates were verified by RT-PCR (Dong et al., 2014). In this study, additional 43 sRNA from 109 remaining candidates were detected by RT-PCR and the role of all verified sRNAs in virulence of Brucella was examined by over-expression in the wild type strain B. abortus 2308. We identified and characterized one sRNA (BASI74) that significantly changed Brucella virulence in macrophage infection model.

\section{MATERIALS AND METHODS}

\section{Bacteria Strains and Culture Conditions}

We performed a routine cultivation of Escherichia coli strains in Luria-Bertani (LB) broth or on LB agar plates with appropriate antibiotic supplementation, if necessary. The Brucella strains were routinely grown in tryptic soy broth (TSB, BD company) at $37^{\circ} \mathrm{C}$ or on tryptic soy agar medium incubated at $37^{\circ} \mathrm{C}$ under $5 \% \mathrm{CO}_{2}$. Additionally, we added chloramphenicol $(30 \mu \mathrm{g} / \mathrm{mL})$, when we cultured the Brucella strains with chloramphenicol resistance. All of the bacterial strains were stored at $-80^{\circ} \mathrm{C}$ and supplemented with $25 \%$ (v/v) glycerol. In order to determine the expression levels of the BASI74 under different conditions, we cultured B. abortus 2308 in TSB ( $\mathrm{pH} 4.5$ ), TSB (10 mM 2,2'dipyridyl), and BMM (Brucella minimum medium)for $4 \mathrm{~h}$ or in TSB $\left(2.5 \mathrm{mM} \mathrm{H}_{2} \mathrm{O}_{2}\right)$ for $30 \mathrm{~min}$.

\section{Mice and Ethics Statement}

Female 4- to 6-week-old BALB/c mice were obtained from Beijing Vital River Laboratory Animal Technology Co., Ltd. All animals were handled in strict accordance with the Experimental Animal Regulation Ordinances defined by the China National Science and Technology Commission; the study was approved by the animal ethics committee of China Institute of Veterinary Drug Control.

\section{RNA Isolation and Reverse Transcription Polymerase Chain Reaction}

We extracted the total RNA of B. abortus 2308 under different stress conditions and different growth stages using Bacterial RNA Kit (Omega) and reverse-transcribed into cDNA using random primers, as previously described (Liu et al., 2012). We performed RT-PCR to verify the expression of the sRNA candidates. $1 \mu \mathrm{l}$ of cDNA sample (without dilution) or total RNA (negative control) was used as template for the PCR. The specific primers of BASI74 used for RT-PCR are listed in Supplementary Table S1. We analyzed the PCR products using a $2 \%$ agarose gel by electrophoresis, and the bands with the appropriate sizes were cut and sequenced by the Beijing Genomics Institute (Shenzhen, China).

\section{Construction of Small RNA Over-expression Strains}

Each putative sRNA encoding sequence (containing the predicted sRNA sequence, about $300 \mathrm{nt}$ upstream and $300 \mathrm{nt}$ downstream sequences) inserted into pBBR1-MCS6 was analyzed to make sure it contains a putative promoter sequence. The constructed over-expression plasmids were verified by sequencing. For construction of sRNA over-expression strains, the pBBR1MCS6 plasmid with putative sRNA encoding sequence was electroporated into $B$. abortus 2308, and then cells were plated onto TSA containing chloramphenicol for selection of positive clones. In addition, the over-expression strains were further verified by PCR using universal primers.

\section{Construction of BASI74 Deletion Mutant}

Construction of recombinant plasmid and selection of marked deletion mutant were performed as previously reported (Zhang et al., 2009). The primers used to construct the recombinant plasmid were listed in Supplementary Table S1.

\section{Quantitative RT-PCR}

In order to detect the expression levels of the sRNAs under different stress conditions described above, we performed RTqPCR as previously described (Dong et al., 2013). Samples were run in triplicate and amplified in a $20 \mu \mathrm{l}$ reaction system containing $10 \mu \mathrm{l} 2 \times$ SYBR $^{\circledR}$ Premix Ex TaqTM II(TAKARA), $100 \mathrm{nM}$ forward and reverse primers, and $1 \mu \mathrm{l}$ appropriately diluted cDNA sample. Primers used for RT-qPCR are provided in Supplementary Table S1. 16S rRNA, expression of which is relatively constant in bacteria, was used as a reference gene.

\section{Cellular Infections}

To investigate intracellular survival of the pathogen, we evaluated the multiplication of $B$. abortus 2308 and its derived strains in J774A.1 murine macrophages. The assays were performed as previously described (Zhang et al., 2009). 


\section{Mouse Infections}

Mice were inoculated intraperitoneally with $100 \mu \mathrm{l}\left(10^{5} \mathrm{CFU}\right)$ of 2308-BASI74 and the parental strain B. abortus 2308. Five mice of one group were euthanized via carbon dioxide asphyxiation at 1 and 4 weeks post-infection. At each time point, the spleens were harvested, weighed, and then homogenized in $1 \mathrm{ml}$ of peptone saline. Serial dilutions were prepared, and 100- $\mu$ l aliquots of each dilution (including the undiluted organ) were plated in duplicate onto TSA plates or TSA plates with $30 \mu \mathrm{g} / \mathrm{mL}$ chloramphenicol (Zhang et al., 2009).

\section{Stress Assays}

We performed the stress response assays as previously reported with slight modifications as following: the Brucella strains derived from a single clone were grown for $48 \mathrm{~h}$ in $4 \mathrm{ml}$ TSB medium. The bacterial cells (initial density of $1 \times 10^{6} \mathrm{CFU} / \mathrm{ml}$ ) were grown in $\mathrm{BMM}$ at $37^{\circ} \mathrm{C}$ with continuous shaking. The concentration of bacteria was measured every 2 days. The number of colony forming units per milliliter was obtained by plating a series of 1:10 dilutions on TSA plates.

To test if over-expression of BASI74 affected bacterial survival under acidic environments, the Brucella strains (with an initial density of $1 \times 10^{7} \mathrm{CFU} / \mathrm{ml}$ ) were cultured in TSB ( $\left.\mathrm{pH} 4.5\right)$ and the concentration of bacteria was measured at $2 \mathrm{~h}$ and $9 \mathrm{~h}$ postinoculation.

In order to determine if over-expression of BASI74 affected bacterial survival under oxidation stress, bacterial strains were adjusted to a concentration of $1 \times 10^{9} \mathrm{CFU} / \mathrm{ml}$, and $100 \mu \mathrm{l}$ of each bacterial strains were seeded on a TSA plate, with a $5.5 \mathrm{~mm}$ sterile filter paper disk in the center of each plate. We placed $10 \mu \mathrm{l}$ of a $30 \%$ solution of $\mathrm{H}_{2} \mathrm{O}_{2}$ onto each disk and incubated at $37^{\circ} \mathrm{C}$ with $5 \% \mathrm{CO}_{2}$. After $72 \mathrm{~h}$ of incubation, the zones of inhibition around each disk were measured.

In order to detect if over-expression of BASI74 affected the iron utilization, we cultured the Brucella strains in an iron limitation medium (TSB with $2.5,5$, and $10 \mathrm{mM} 2,2^{\prime}$-dipyridyl) for $48 \mathrm{~h}$. The bacteria were cultured in this medium at the same initial density $\left(1 \times 10^{6} \mathrm{CFU} / \mathrm{ml}\right)$, and we then determined the CFUs at $48 \mathrm{~h}$ for each strain.

\section{Bioinformatics Data Analysis}

To determine the position of putative promoter sequence, the upstream sequences of each verified sRNAs were analyzed using BDGP: Neural Network Promoter Prediction ${ }^{1}$, with the parameters for the software set at their default settings.

We predicted the target genes for the sRNA using CopraRNA ${ }^{2}$, with the parameters for the software set at their default settings (Wright et al., 2009).

\section{Verification of the Target Gene Regulated by BASI74 and $\beta$-Galactosidase Assays}

The E. coli-based reporter system used for verification of genes regulated by BASI74 was constructed as previously described

${ }^{1}$ http://www.fruitfly.org/seq_tools/promoter.html

${ }^{2} \mathrm{http} / / /$ rna.informatik.uni-freiburg.de
(Dong et al., 2014). The primers used to amplify BASI74 and the putative target sequences are listed in Supplementary Table S1, and the plasmids used in this study can be found in Supplementary Table S2.

\section{Statistical Analysis}

Differences between the means of the experimental and control groups were analyzed using the independent samples $t$-test

TABLE 1 | Verified sRNA in this work.

\begin{tabular}{|c|c|c|c|}
\hline sRNA Name & sRNA start & end & Length of sRNA (nt) \\
\hline BAS I 365 & 24475 & 24577 & 102 \\
\hline BAS I 371 & 29595 & 29988 & 393 \\
\hline BAS I 387 & 47061 & 47307 & 246 \\
\hline BAS I 22 & 100793 & 100872 & 79 \\
\hline BAS I 23 & 100828 & 100944 & 116 \\
\hline BAS I 262 & 128868 & 129114 & 246 \\
\hline BAS I 35 & 254741 & 254855 & 114 \\
\hline BAS I 62 & 521937 & 522023 & 86 \\
\hline BAS I 74 & 713147 & 713233 & 86 \\
\hline BAS I 84 & 815539 & 815617 & 78 \\
\hline BAS I 244 & 1098391 & 1098472 & 81 \\
\hline BAS I 245 & 1099846 & 1100145 & 299 \\
\hline BAS I 122 & 1173218 & 1173318 & 100 \\
\hline BAS I 130 & 1221129 & 1221226 & 97 \\
\hline BAS I 133 & 1249641 & 1249811 & 170 \\
\hline BAS | 137 & 1289949 & 1290101 & 152 \\
\hline BAS I 151 & 1441442 & 1441521 & 79 \\
\hline BAS I 273 & 1445660 & 1445799 & 139 \\
\hline BAS I 9 & 1490038 & 1490143 & 105 \\
\hline BAS I 283 & 1500367 & 1500491 & 124 \\
\hline BAS I 176 & 1603445 & 1603565 & 120 \\
\hline BAS I 304 & 1648877 & 1649137 & 260 \\
\hline BAS I 306 & 1662860 & 1663033 & 173 \\
\hline BAS I 193 & 1688162 & 1688286 & 124 \\
\hline BAS I 214 & 1971684 & 1971765 & 81 \\
\hline BAS I 344 & 2005699 & 2006063 & 364 \\
\hline BAS I 345 & 2017052 & 2017142 & 90 \\
\hline BAS I 218 & 2032657 & 2032746 & 89 \\
\hline BAS I 221 & 2056362 & 2056509 & 147 \\
\hline BAS I 228 & 2084505 & 2084582 & 77 \\
\hline BAS II 152 & 873615 & 873740 & 125 \\
\hline BAS $\| 36$ & 295058 & 295164 & 106 \\
\hline BAS $\| 47$ & 433815 & 433936 & 121 \\
\hline BAS II 149 & 75638 & 75764 & 126 \\
\hline BAS $\| 37$ & 309649 & 309775 & 126 \\
\hline BAS ॥ 99 & 1099166 & 1099368 & 202 \\
\hline BAS ॥ 39 & 325182 & 325397 & 215 \\
\hline BAS II 133 & 508838 & 509001 & 163 \\
\hline BAS $\| 5$ & 580622 & 580840 & 218 \\
\hline BAS $\| 73$ & 824439 & 824525 & 86 \\
\hline BAS II 150 & 824439 & 824613 & 174 \\
\hline BAS $\| 74$ & 824439 & 824637 & 198 \\
\hline BAS \| 117 & 381044 & 381193 & 149 \\
\hline
\end{tabular}


included in the program SPSS 17.0. Differences were considered significant at $p$-values of $<0.05$.

\section{RESULTS}

\section{Identification of Additional 43 sRNAs Expressed in Brucella abortus 2308}

Our previous studies had identified 129 sRNAs candidates of Brucella using bioinformatics methods, and 7 of 20 tested sRNA candidates were verified to be present (Dong et al., 2014). In this study, we extracted the total RNA of B. abortus strain 2308 and detected if the remaining 109 sRNA candidates were expressed using RT-PCR. A total of 43 sRNAs could be detected by RT-PCR and sequencing (Table 1), out of which the RT-PCR result of 24 sRNAs were shown in Figure 1.

\section{Identification of sRNA Over-expressed Strains With Reduced Survival Compared With Parental Strain in Macrophages}

In this study, several cis-encoded sRNAs were verified, and it was impossible to construct mutants of cis-encoded sRNAs without affecting their neighboring target genes. To address this problem, we over-expressed all the 43 verified sRNAs in the wild type Brucella strain, and detected if the virulence of these over-expressed strains were altered.
Overall, the virulence of 42 sRNA over-expression strains were almost equivalent to that of 2308 and 2308-pBBR1, while over-expression of the sRNA BASI74(named 2308-BASI74) significantly reduced Brucella virulence in the macrophage infection model at $48 \mathrm{~h}$ post-infection $(p<0.01)$ (Table 2$)$.

\section{Over-expression of BASI74 Affected the Virulence of $B$. abortus 2308}

To further confirm the relationship between BASI74 and reduced survival ability in macrophages, a BASI74-deletion strain(named $\triangle$ BASI74) was constructed and the virulence of 2308-BASI74 and $\triangle$ BASI74 in J774A.1 macrophages was detected at different time points.

Before the macrophage infection assay, the expression of BASI74 was detected in both 2308-BASI74 and $\triangle$ BASI74. The results of RT-qPCR showed that the expression levels of BASI74 were not significantly different between 2308 and $\triangle$ BASI74, while that of 2308-BASI74 was about 8 -fold higher than that of 2308(Supplementary Table S3). According to the blast result of BASI74 sequence in B. abortus 2308, several highly homologous sequences were found in both chromosomes, I and II (Supplementary Table S4).

As shown in Figure 2, the intracellular bacteria load of 2308BASI74 was significantly reduced at $48 \mathrm{~h}$ post-infection compared to that of 2308 and 2308-PBBR1 $(p<0.01)$. However, the survival ratio of $\triangle B A S I 74$ showed no difference, compared with that of 2308 at each time point.
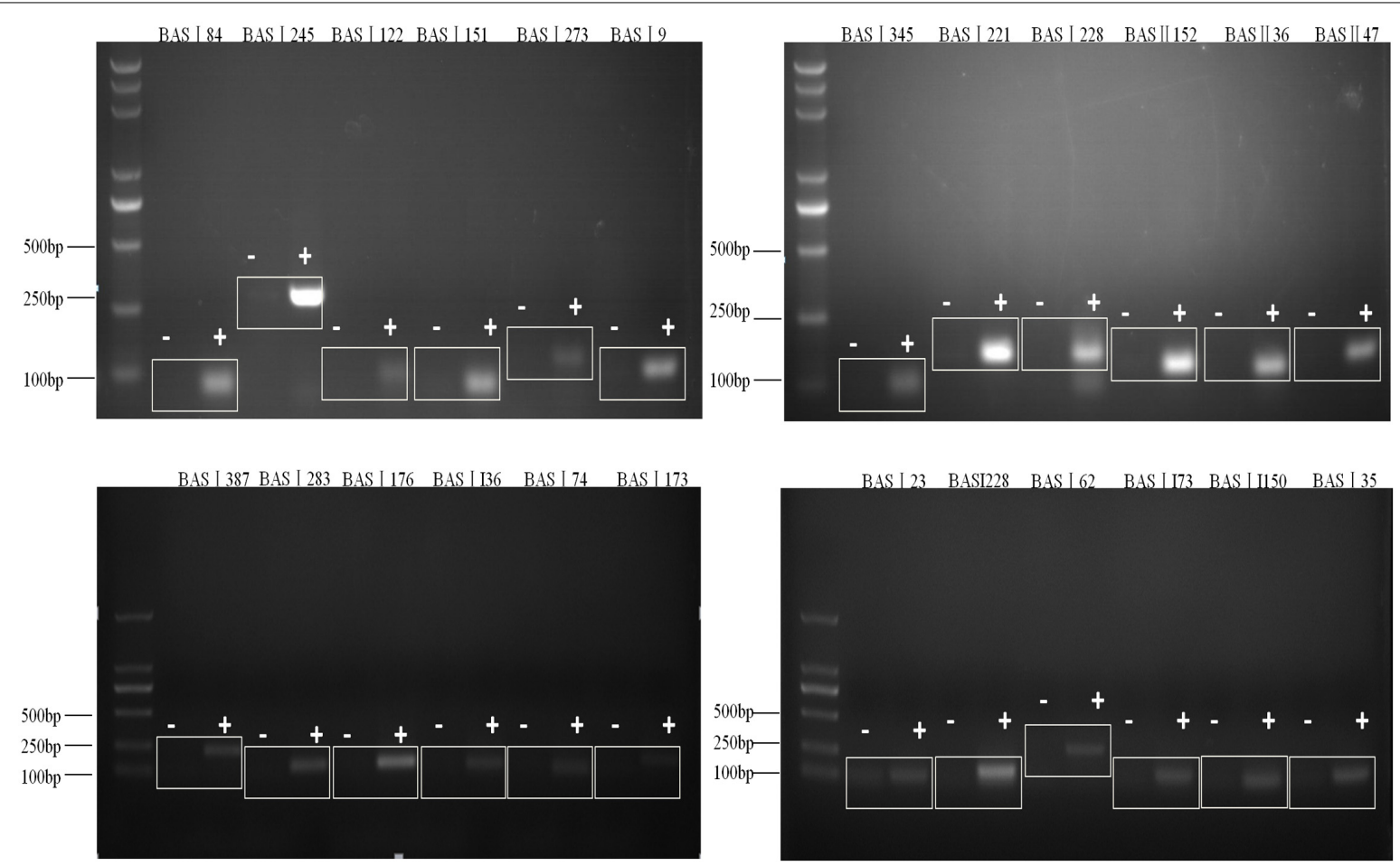

FIGURE 1 | Verification of SRNAs in B. abortus 2308. RT-PCR verification of the transcriptional unit of SRNA candidates. RNA prepared from B. abortus 2308 grown to stationary phase at $37^{\circ} \mathrm{C}$ was used for RT-PCR. The regions to be amplified were shown by bars with numbers. " + " represents reactions with reverse transcriptase and "-" represents reactions without reverse transcriptase. 
TABLE 2 | Multiplication ability of sRNA over expression strains in J774A.1 macrophages.

\begin{tabular}{|c|c|c|c|c|c|c|c|c|c|}
\hline $\begin{array}{l}\text { sRNA } \\
\text { Name }\end{array}$ & $1 \mathrm{~h} \mathrm{CFU}$ & $\begin{array}{c}\text { Over- } \\
\text { expression } \\
\text { strain/WT(1 h) }\end{array}$ & 48 h CFU & $\begin{array}{c}\text { Over- } \\
\text { expression } \\
\text { strain/WT(48 h) }\end{array}$ & $\begin{array}{l}\text { sRNA } \\
\text { Name }\end{array}$ & $1 \mathrm{~h} \mathrm{CFU}$ & $\begin{array}{c}\text { Over- } \\
\text { expression } \\
\text { strain/WT(1 h) }\end{array}$ & 48 h CFU & $\begin{array}{c}\text { Over- } \\
\text { expression } \\
\text { strain/WT } \\
\text { (48 h) }\end{array}$ \\
\hline BAS I 74 & $1.98 E+03$ & $33.51 \%$ & $9.17 \mathrm{E}+03$ & $0.61 \%$ & BAS I 306 & $8.42 E+02$ & $14.23 \%$ & $4.98 E+05$ & $33.40 \%$ \\
\hline BAS I 22 & $9.67 E+02$ & $16.34 \%$ & $4.05 E+05$ & $27.14 \%$ & BAS I 344 & $8.66 E+03$ & $146.32 \%$ & $8.61 E+05$ & $57.70 \%$ \\
\hline BAS I 23 & $8.33 E+02$ & $14.08 \%$ & $2.45 E+05$ & $16.42 \%$ & BAS I 345 & $8.42 \mathrm{E}+02$ & $14.23 \%$ & $2.28 \mathrm{E}+05$ & $15.30 \%$ \\
\hline BAS I 35 & $1.28 \mathrm{E}+03$ & $21.55 \%$ & $1.23 E+05$ & $8.27 \%$ & BAS I 365 & $2.24 \mathrm{E}+03$ & $37.89 \%$ & $4.16 \mathrm{E}+05$ & $27.87 \%$ \\
\hline BAS I 62 & $2.71 E+03$ & $45.77 \%$ & $2.62 E+05$ & $17.54 \%$ & BAS I 371 & $9.33 E+02$ & $15.77 \%$ & $5.83 E+05$ & $39.04 \%$ \\
\hline BAS I 9 & $8.33 E+02$ & $14.08 \%$ & $7.52 E+05$ & $50.38 \%$ & BAS I 387 & $5.42 E+03$ & $91.55 \%$ & $1.02 E+06$ & $68.16 \%$ \\
\hline BAS I 84 & $2.80 E+03$ & $47.32 \%$ & $4.33 E+05$ & $29.04 \%$ & BAS $\| 5$ & $2.01 E+03$ & $33.94 \%$ & $3.66 \mathrm{E}+05$ & $24.52 \%$ \\
\hline BAS I 122 & $1.10 E+03$ & $18.59 \%$ & $1.15 E+06$ & $77.08 \%$ & BAS II 36 & $8.42 E+02$ & $14.23 \%$ & $3.18 E+05$ & $21.34 \%$ \\
\hline BAS I 130 & $8.67 E+03$ & $146.48 \%$ & $1.10 E+06$ & $73.39 \%$ & BAS ॥ 37 & $1.15 E+03$ & $19.44 \%$ & $6.55 E+05$ & $43.90 \%$ \\
\hline BAS I 133 & $1.63 E+03$ & $27.46 \%$ & $5.52 E+05$ & $36.97 \%$ & BAS ॥ 39 & $1.57 E+03$ & $26.48 \%$ & $3.68 E+05$ & $24.69 \%$ \\
\hline BAS I 137 & $3.11 E+03$ & $52.53 \%$ & $8.50 E+05$ & $56.97 \%$ & BAS \| 47 & 1.99E + 03 & $33.67 \%$ & $4.15 E+05$ & $27.81 \%$ \\
\hline BAS I 151 & $1.91 E+03$ & $32.25 \%$ & $7.80 E+05$ & $52.28 \%$ & BAS $\| 73$ & $9.08 E+02$ & $15.35 \%$ & $3.22 E+05$ & $21.56 \%$ \\
\hline BAS I 176 & $2.28 \mathrm{E}+03$ & $38.45 \%$ & $2.84 \mathrm{E}+05$ & $19.05 \%$ & BAS II 74 & $1.15 E+03$ & $19.49 \%$ & $6.33 E+05$ & $42.45 \%$ \\
\hline BAS I 193 & $1.49 E+03$ & $25.22 \%$ & $5.88 E+05$ & $39.43 \%$ & BAS ॥ 99 & $1.60 E+03$ & $27.04 \%$ & $3.33 E+05$ & $22.34 \%$ \\
\hline BAS I 214 & $2.31 E+03$ & $39.01 \%$ & $5.47 E+05$ & $36.64 \%$ & BAS ॥ 117 & $5.42 E+03$ & $91.55 \%$ & $6.23 E+05$ & $41.78 \%$ \\
\hline BAS I 218 & $1.79 E+03$ & $30.29 \%$ & $5.58 E+05$ & $37.42 \%$ & BAS \|I 133 & $3.27 E+03$ & $55.21 \%$ & $9.51 E+05$ & $63.73 \%$ \\
\hline BAS I 221 & $8.33 E+03$ & $140.83 \%$ & $1.25 E+06$ & $83.44 \%$ & BAS ॥ 149 & $1.50 E+03$ & $25.35 \%$ & $4.96 E+05$ & $33.23 \%$ \\
\hline BAS I 228 & $1.81 E+03$ & $30.56 \%$ & $1.00 E+06$ & $67.02 \%$ & BAS II 152 & $2.90 \mathrm{E}+03$ & $49.01 \%$ & $8.02 E+05$ & $53.73 \%$ \\
\hline BAS I 244 & $5.50 E+03$ & $92.95 \%$ & $9.47 E+05$ & $63.45 \%$ & BAS ॥ 150 & $1.50 E+03$ & $25.35 \%$ & $4.96 E+05$ & $33.23 \%$ \\
\hline BAS I 245 & $1.03 E+03$ & $17.46 \%$ & $8.27 E+05$ & $55.41 \%$ & $\begin{array}{l}\text { 2308- } \\
\text { pBBR1 }\end{array}$ & $2.14 \mathrm{E}+03$ & $36.20 \%$ & $1.00 E+06$ & $67.09 \%$ \\
\hline BAS I 262 & $9.25 E+02$ & $15.63 \%$ & $3.97 E+05$ & $26.59 \%$ & 2308 & $5.92 E+03$ & $100.00 \%$ & $1.49 E+06$ & $100.00 \%$ \\
\hline BAS I 273 & $8.42 E+02$ & $14.23 \%$ & $3.95 E+05$ & $26.47 \%$ & & & & & \\
\hline BAS I 283 & $7.08 E+03$ & $119.71 \%$ & $5.17 E+05$ & $34.63 \%$ & & & & & \\
\hline BAS I 304 & $1.28 \mathrm{E}+03$ & $21.68 \%$ & $4.19 E+05$ & $28.09 \%$ & & & & & \\
\hline
\end{tabular}

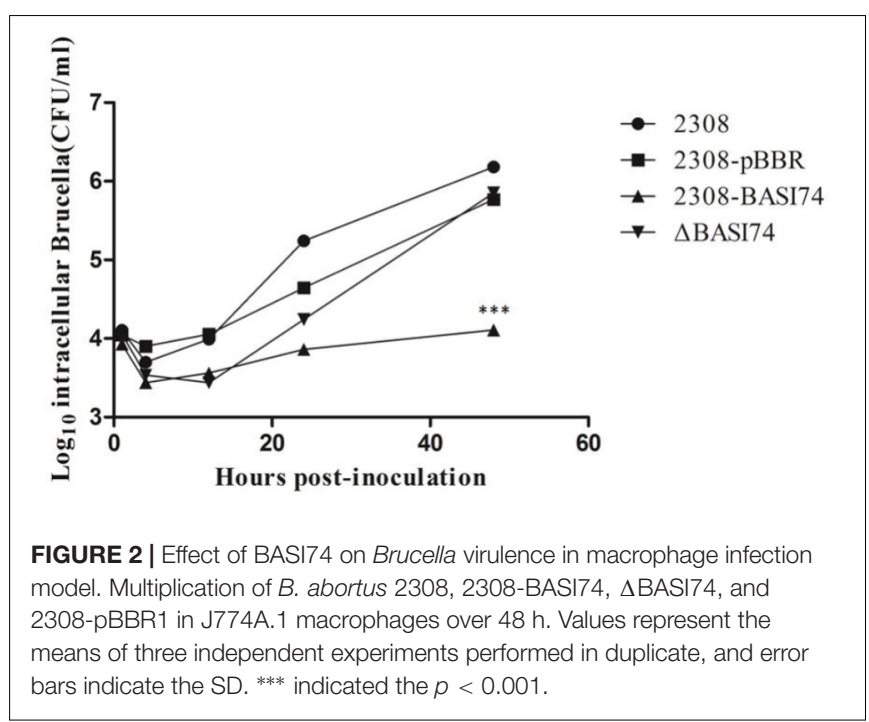

To evaluate the virulence in vivo, $\mathrm{BALB} / \mathrm{c}$ mice were infected with both 2308-BASI 74 and B. abortus 2308. Compared with the parental strain 2308, the spleen weight of 2308-BASI74 infected mice was significantly lighter at both $1 \operatorname{week}(p<0.01)$ and 4 weeks $(p<0.001)$ post-infection (Figure 3A), while no significant difference was observed in the splenic CFUs between 2308-BASI74 and 2308-infected groups at each time $\operatorname{point}(p>0.05)$ (Figure 3B).

\section{Expression Pattern of BASI74 in B. abortus 2308}

RT-qPCR with RNA samples isolated from the bacteria grown under different stress conditions or harvested at different stages was performed in order to characterize the expression pattern of BASI74. We found that BASI74 was produced at all growth phases, and the expression level increased to the peak at $8 \mathrm{~h}$ postincubation (Figure 4A). The expression levels of BASI74 were not significantly changed under iron deficiency $(10 \mathrm{mM} \mathrm{2,2}$ dipyridyl for $4 \mathrm{~h}$ ) or oxidative ( $2.5 \mathrm{mM} \mathrm{H}_{2} \mathrm{O}_{2}$ for $30 \mathrm{~min}$ ) stress, or even in BMM compared with in normal TSB culture. However the level of BASI74 increased more than 4 -fold under acidic $(\mathrm{pH}$ 4.5 for $4 \mathrm{~h}$ ) stress than in normal TSB control (Figure 4B).

\section{The BASI74 Was Involved in Stress Responses}

The characteristics of 2308-BASI74 in macrophages promoted us to study the underlying mechanisms. Previous studies have demonstrated that many sRNAs are related to stress response, and 

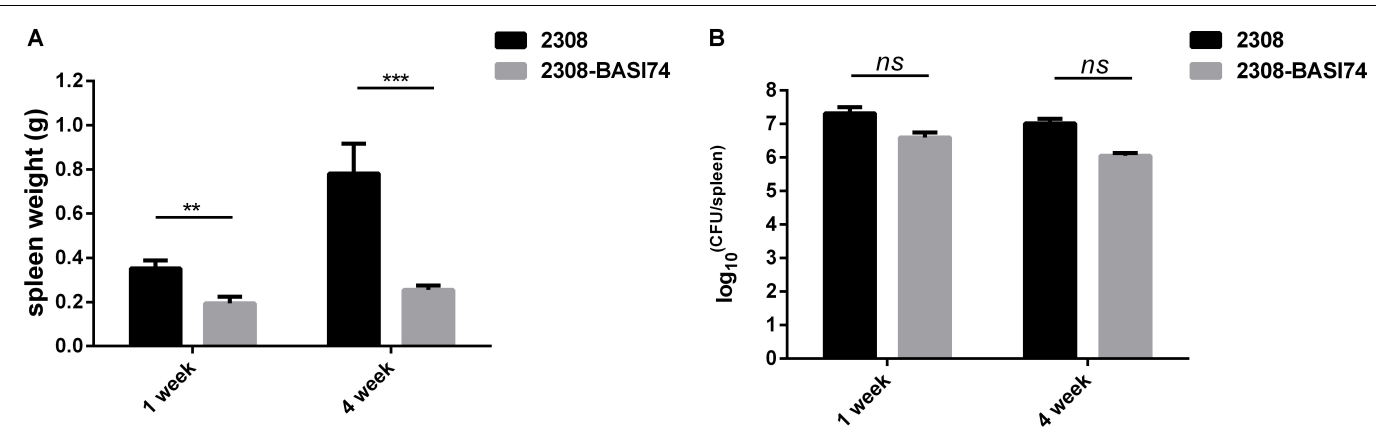

FIGURE 3 | Effect of BASI74 on Brucella virulence in mice infection model. (A) The spleen weight of 2308-BASI74 and 2308 infected mice at 1 and 4 weeks post infection. (B) The splenic CFUs of 2308-BASI74 and 2308 in infected mice at 1 and 4 weeks post infection. ${ }^{* *}$ indicated the $p<0.01 . * * *$ indicated the $p<0.001$.

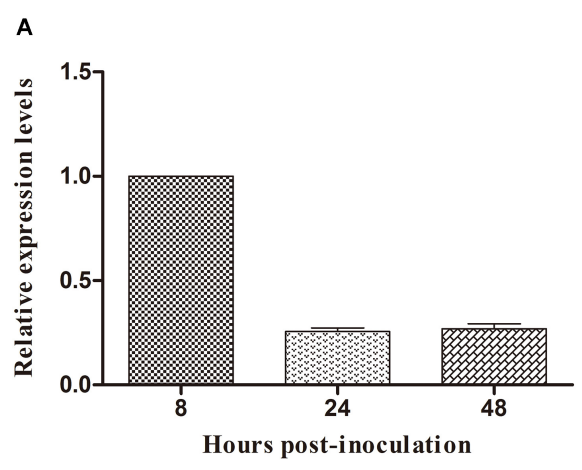

B

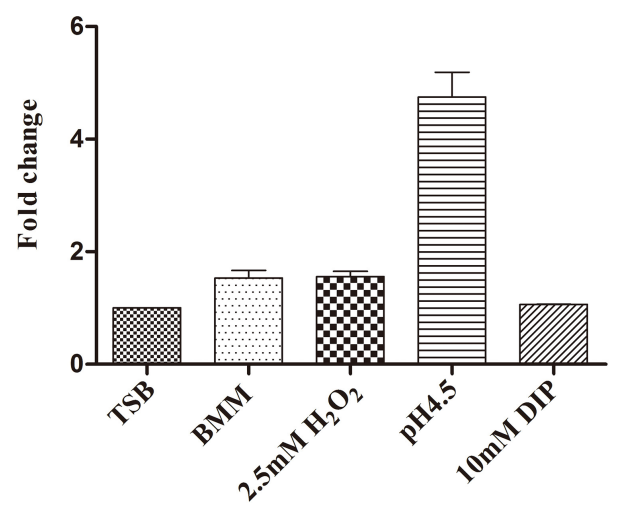

FIGURE 4 | Expression pattern of BASI74 in B. abortus 2308. (A) Expression levels of BASI74 at different growth stages in TSB medium. (B) Expression levels of BASI74 under different stress conditions. B. abortus 2308 from a single clone was grown for $48 \mathrm{~h}$ in TSB medium and then cultured in TSB $(\mathrm{pH}$ 4.5), TSB + 10 mM 2,2'-dipyridyl, BMM (Brucella minimum medium) for $4 \mathrm{~h}$ or in TSB $+2.5 \mathrm{mM} \mathrm{H}_{2} \mathrm{O}_{2}$ for $30 \mathrm{~min}$. The expression levels of BASI74 under different stress conditions were compared to samples cultured in TSB medium. DIP stands for 2,2'-dipyridyl. therefore the survival ability of the over-expression strains under different stress conditions was tested.

As shown in Figure 5A, the survival ratio of 2308-BASI74 cultured in an acidic medium for $9 \mathrm{~h}$ was almost the same as that of 2308-pBBR1 and 2308. Neither did we find significant differences of growth ratio among these three strains in the $\mathrm{H}_{2} \mathrm{O}_{2}$ disk sensitivity assays (Figure 5B). In BMM culture, the growth ratio of 2308-BASI74 gradually deviated since 4 days post-incubation compared with that of 2308 and 2308-pBBR1 cells, and turned out to be significantly lower at 8 days postincubation $(p<0.05)$ (Figure 5C). In addition, the survival ratio of the 2308-BASI74 was much lower than that of 2308 and 2308-pBBR1 when cultured in iron limited TSB (10 mM $2,2^{\prime}$-dipyridyl) for $48 \mathrm{~h}$ (Figure 5D). These data revealed that BASI74 was involved in growth in iron-limiting medium and BMM.

\section{Identification of Targets Regulated by BASI74}

To identify the genes regulated by the BASI74 RNA, we performed an in silico analysis with sTarPicker (see footnote 2).

As shown in Table 3, for BAB1_1361, BAB1_1335, the $\beta$-galactosidase activity of the strains containing the combination of the sRNA-encoding plasmid and target lacZ fusion plasmids were significantly reduced compared with the vector and lac $Z$ fusion plasmids combination group. On the contrary, co-expression of BASI74 with the 5'-UTR of BAB1_1154 or BAB1_0847 lacZ fusion plasmids significantly increased the $\beta$-galactosidase activity. For BAB1_0097 and BAB1_0343, no obvious difference was observed between the BASI74 and vector group.. Except for that of BAB1_1154 (encoding cytosine-N4specific DNA methyltransferase), functions of all other three targets were still unknown.

To further determine whether these targets were regulated by BASI74, the expression level of four putative targets was tested by RT-qPCR in both $\triangle$ BASI74 and 2308-BASI74. As shown in Table 4, the transcriptional level of all four verified targets was upregulated in 2308-BASI74, while none of the four targets was affected in the $\triangle$ BASI 74 .

\section{DISCUSSION}

Previous studies have demonstrated that sRNAs were related with the proper expression of virulence factors in a variety of 

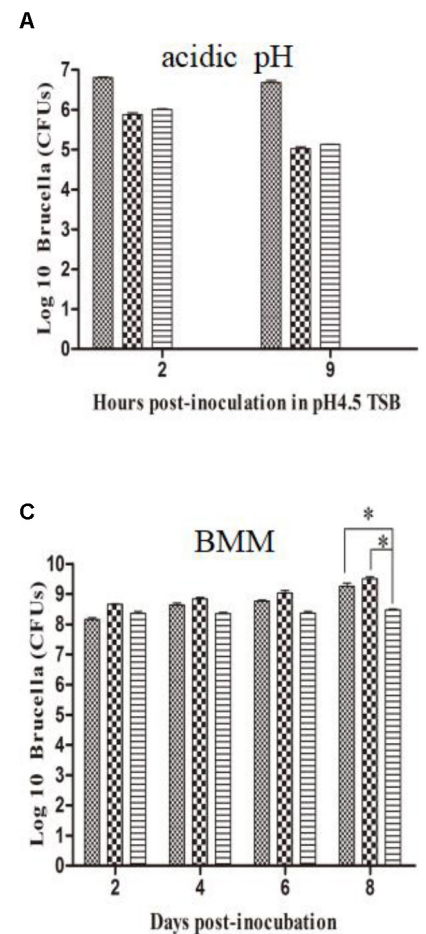

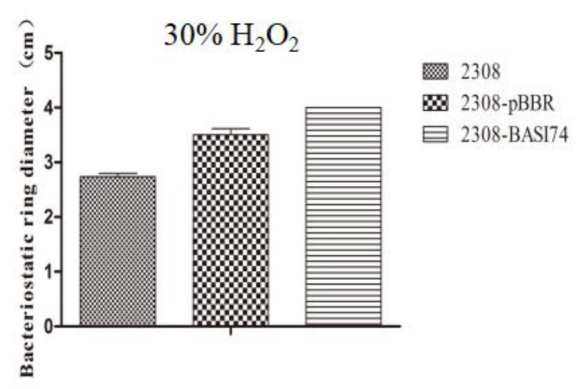

D

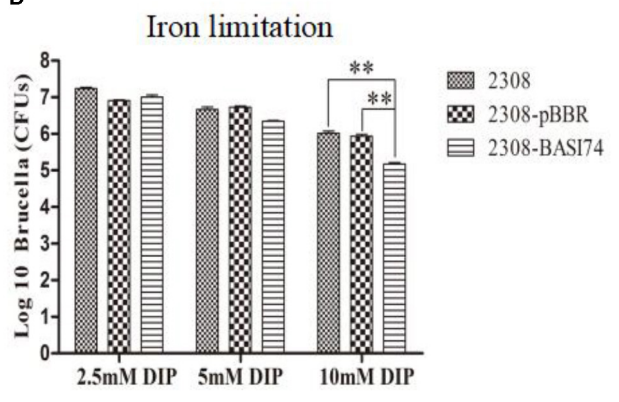

FIGURE 5 | Growth behavior of strains over-producing BASI74 under different stress conditions. (A) Over-expression of BASI74 did not affect the survival ratio of B. abortus in acidic medium (TSB pH 4.5). (B) The 2308-BASI74 strain had equal sensitivity to $30 \% \mathrm{H}_{2} \mathrm{O}_{2}$ compared to two control strains. (C) BASI74 affected bacteria growth in Brucella minimum medium. (D) Over-expression of BASI74 reduced the survival ratio of $B$. abortus under iron-limiting condition. Values represent the means of three independent experiments, and error bars indicate the SD. ${ }^{*}$ indicated the $p<0.05$. ${ }^{*}$ indicated the $p<0.01$.

TABLE 3 | Verification of the interaction between BASI74 and putative target sequences.

\begin{tabular}{|c|c|c|c|c|}
\hline Putative target genes & \multicolumn{2}{|c|}{$\beta$ - galactosidase activity (Miller units) } & Fold change BASI74/vector & $p$-value \\
\hline BAB1_0097 & $9.21 \pm 2.23$ & $11.61 \pm 2.71$ & 1.26 & 0.8093 \\
\hline BAB1_0343 & $3.15 \pm 1.21$ & $2.01 \pm 0.83$ & 0.64 & 0.1253 \\
\hline BAB1_0847 & $15.57 \pm 2.77$ & $23.65 \pm 2.57$ & 1.52 & $<0.05$ \\
\hline BAB1_1154 & $14.99 \pm 2.41$ & $47.18 \pm 1.935$ & 3.15 & $<0.001$ \\
\hline
\end{tabular}

The data were expressed as averages \pm standard deviations (SD). Three independent experiments were performed.

pathogenic bacteria (Papenfort and Vogel, 2010), and several recent studies also showed that sRNAs directly correlated with the virulence of organisms such as Listeria (Mraheil et al., 2011),

TABLE 4 | The transcriptional levels of four verified target genes in 2308-BASI74 and $\triangle \mathrm{BASI}$. 4 .

\begin{tabular}{lcc}
\hline Gene & \multicolumn{2}{c}{ Fold change } \\
\cline { 2 - 3 } & 2308-BASI74 vs. 2308 & DBASI74 vs. 2308 \\
\hline BAB1_1361 & 4.52 & 1.32 \\
BAB1_1335 & 2.69 & 1.17 \\
BAB1_1154 & 7.89 & 0.93 \\
BAB1_0847 & 3.89 & 0.77
\end{tabular}

Salmonella (Gong et al., 2011), Vibrio (Song et al., 2008), Yersinia (Koo et al., 2011), and Brucella (Caswell et al., 2012).

In this study, it was interesting to find that over-expression of BASI74 locus reduced Brucella virulence in macrophages, while deletion of putative BASI74 encoding sequence did not affect Brucella virulence. The results of RT-qPCR showed that the transcriptional level of BASI74 between 2308 and $\triangle$ BASI74 were not significantly changed, while that of 2308-BASI74 was about 8 -fold higher than that of 2308, which might possibly explain the difference of virulence between 2308-BASI74 and $\Delta$ BASI74. We speculated that there possibly existed more than one locus encoding BASI74 in the genome of 2308.

Further, we observed a consistent trend in the downstream target genes. The transcriptional level of four verified target genes 
were changed more than 2-fold in 2308-BASI74, while none of the four targets was affected in $\triangle$ BASI74.

In a previous study, it was also demonstrated that overexpression of sRNAs could result in more dramatic effects on their regulated targets than sRNAs deletion (Koo et al., 2011). Our result was consistent with data previously reported.

Taken together, these data indicated that the reason that $\triangle$ BASI74 could not significantly affect the virulence of Brucella strains might be explained by the redundancy in genetic structure and function.

Previous studies demonstrated that AbcR sRNAs had redundant and compensatory functions in B. abortus 2308 (Caswell et al., 2012). In addition, the four Qrr sRNAs involved in the regulation of quorum sensing are redundant in Vibrio cholerae (Lenz et al., 2004). In our study, the probable multiple copies of BASI74 might indicate the important role of this sRNA, and the redundancy of this sRNA may be an evolutionary adaption ensuring the proper expression of essential genes.

As a facultative intracellular pathogen, B. abortus encounters formidable environmental stresses such as nutrient deprivation during its interactions with the host cells (Roop et al., 2009). In addition, Brucella strains required iron transporters for the expression of wild type virulence in natural and experimental hosts (Roop, 2012). Our results that 2308-BASI74 exhibited lower growth in iron-limiting and nutrient deprivation medium indicated that the attenuation of 2308-BASI74 was probably related with its reduced tolerance under these two types of stresses.

Although 2308-BASI74 was attenuated in macrophage infection model, no significant difference of virulence was observed in the mice infection assay at different time points (Figure 3B). This disagreement of Brucella virulence tested by macrophage and mice infection models was not uncommon. In the study of Brucella quorum sensing regulator BlxR, the $\triangle b l x R$ strain exhibited reduced growth in macrophages, while this mutant was not highly attenuated in mice (Rambow-Larsen et al., 2008). Besides, it was worthy noting that the spleen weight of the mice infected with 2308-BASI74 was significantly lighter than that of 2308 in mice infection models at both 1 and 4 weeks post-infection. This observation, which was also found in the mice infection assay of Brucella attenuated strain, indicated that the 2308-BASI74 might induce a different immune response in the mice infection model.

\section{REFERENCES}

Abdou, E., Deredjian, A., Jimenez, D. B. M., Kohler, S., and Jubier-Maurin, V. (2013). RegA, the regulator of the two-component system RegB/RegA of Brucella suis, is a controller of both oxidative respiration and denitrification required for chronic infection in mice. Infect. Immun. 81, 2053-2061. doi: 10.1128/IAI.00063-13

Brambila-Tapia, A. J., and Perez-Rueda, E. (2014). A functional and phylogenetic comparison of quorum sensing related genes in Brucella melitensis 16M. J. Microbiol. 52, 709-715. doi: 10.1007/s12275-014-3570-x

Caswell, C. C., Gaines, J. M., Ciborowski, P., Smith, D., Borchers, C. H., Roux, C. M., et al. (2012). Identification of two small regulatory RNAs linked to virulence in Brucella abortus 2308. Mol. Microbiol. 85, 345-360. doi: 10.1111/j.1365-2958.2012.08117.x
Previous studies have demonstrated that bacterial DNA methyltransferases were not only associated with restrictionmodification systems, but also with chromosome replication, transcription, repair, and many other fundamental processes (Reisenauer et al., 1999). In addition, some recent studies have demonstrated that DNA adenine methylation play an important role in host-pathogen interactions (Marinus and Casadesus, 2009). In B. abortus, the CcrM DNA methyltransferase was also reported to be essential for viability, and its over-expression attenuated intracellular replication in murine macrophages (Robertson et al., 2000). In Helicobacter pylori, C5-cytosine methylation also affects the expression of several genes related to motility, adhesion, and virulence (Kumar et al., 2012). However, the cytosine-N4-specific DNA methyltransferase can hardly be related with bacterial virulence. As a trans-encoded sRNA, BASI74 could regulate more than one target mRNA. In this study, we only verified the top six putative targets with the highest scores in the prediction result, and more targets of BASI74 needs to be verified in the future. Thus, we hypothesized that overexpression of BASI74 might have various effects on more different targets including the target gene BAB1_1154 encoding DNA methylation.

\section{AUTHOR CONTRIBUTIONS}

HD wrote the paper. XP, YL, TW, and XW performed the experiments. CW and QW conceived and designed the experiments. YD, TH, LY, and JD analyzed the data.

\section{FUNDING}

This work was supported by the National Natural Science Foundation of China (Nos. 31602055 and 31372446) and the National Basic Research Program of China (973 Program) (No. 2010CB530202).

\section{SUPPLEMENTARY MATERIAL}

The Supplementary Material for this article can be found online at: https://www.frontiersin.org/articles/10.3389/fmicb. 2018.02173/full\#supplementary-material

Dong, H., Liu, W., Peng, X., Jing, Z., and Wu, Q. (2013). The effects of MucR on expression of type IV secretion system, quorum sensing system and stress responses in Brucella melitensis. Vet. Microbiol. 166, 535-542. doi: 10.1016/j. vetmic.2013.06.023

Dong, H., Peng, X., Wang, N., and Wu, Q. (2014). Identification of novel sRNAs in Brucella abortus 2308. FEMS Microbiol. Lett. 354, 119-125. doi: 10.1111/15746968.12433

Gong, H., Vu, G. P., Bai, Y., Chan, E., Wu, R., Yang, E., et al. (2011). A Salmonella small non-coding RNA facilitates bacterial invasion and intracellular replication by modulating the expression of virulence factors. PLoS Pathog. 7:e1002120. doi: 10.1371/journal.ppat.1002120

Koo, J. T., Alleyne, T. M., Schiano, C. A., Jafari, N., and Lathem, W. W. (2011). Global discovery of small RNAs in Yersinia pseudotuberculosis identifies Yersinia-specific small, noncoding RNAs required for virulence. 
Proc. Natl. Acad. Sci. U.S.A. 108, E709-E717. doi: 10.1073/pnas.11016 55108

Kovach, M. E., Phillips, R. W., Elzer, P. H., Roop, R. M. II, and Peterson, K. M. (1994). pBBR1MCS: a broad-host-range cloning vector. Biotechniques 16, $800-802$.

Kumar, R., Mukhopadhyay, A. K., Ghosh, P., and Rao, D. N. (2012). Comparative transcriptomics of $\mathrm{H}$. pylori strains AM5, SS1 and their hpyAVIBM deletion mutants: possible roles of cytosine methylation. PLoS One 7:e42303. doi: 10.1371/journal.pone.0042303

Lenz, D. H., Mok, K. C., Lilley, B. N., Kulkarni, R. V., Wingreen, N. S., and Bassler, B. L. (2004). The small RNA chaperone Hfq and multiple small RNAs control quorum sensing in Vibrio harveyi and Vibrio cholerae. Cell 118, 69-82. doi: 10.1016/j.cell.2004.06.009

Liu, W., Dong, H., Liu, W., Gao, X., Zhang, C., and Wu, Q. (2012). OtpR regulated the growth, cell morphology of $B$. melitensis and tolerance to beta-lactam agents. Vet. Microbiol. 159, 90-98. doi: 10.1016/j.vetmic.2012. 03.022

Marinus, M. G., and Casadesus, J. (2009). Roles of DNA adenine methylation in host-pathogen interactions: mismatch repair, transcriptional regulation, and more. FEMS Microbiol. Rev. 33, 488-503. doi: 10.1111/j.1574-6976.2008. 00159.x

Mraheil, M. A., Billion, A., Mohamed, W., Mukherjee, K., Kuenne, C., Pischimarov, J., et al. (2011). The intracellular sRNA transcriptome of Listeria monocytogenes during growth in macrophages. Nucleic Acids Res. 39, 4235-4248. doi: 10.1093/nar/gkr033

Papenfort, K., and Vogel, J. (2010). Regulatory RNA in bacterial pathogens. Cell Host Microbe 8, 116-127. doi: 10.1016/j.chom.2010.06.008

Rambow-Larsen, A. A., Rajashekara, G., Petersen, E., and Splitter, G. (2008). Putative quorum-sensing regulator BlxR of Brucella melitensis regulates virulence factors including the type IV secretion system and flagella. J. Bacteriol. 190, 3274-3282. doi: 10.1128/JB.01915-07

Reisenauer, A., Kahng, L. S., McCollum, S., and Shapiro, L. (1999). Bacterial DNA methylation: a cell cycle regulator? J. Bacteriol. 181, 5135-5139.

Robertson, G. T., Reisenauer, A., Wright, R., Jensen, R. B., Jensen, A., Shapiro, L., et al. (2000). The Brucella abortus CcrM DNA methyltransferase is essential for viability, and its overexpression attenuates intracellular replication in murine macrophages. J. Bacteriol. 182, 3482-3489. doi: 10.1128/JB.182.12.3482-3489. 2000

Roop, R. N. (2012). Metal acquisition and virulence in Brucella. Anim. Health Res. Rev. 13, 10-20. doi: 10.1017/S1466252312000047
Roop, R. N., Gaines, J. M., Anderson, E. S., Caswell, C. C., and Martin, D. W. (2009). Survival of the fittest: how Brucella strains adapt to their intracellular niche in the host. Med. Microbiol. Immunol. 198, 221-238. doi: 10.1007/s00430009-0123-8

Sheehan, L. M., and Caswell, C. C. (2017). A 6-nucleotide regulatory motif within the AbcR small RNAs of Brucella abortus mediates host-pathogen interactions. mBio 8:e00473-17. doi: 10.1128/mBio.00473-17

Song, T., Mika, F., Lindmark, B., Liu, Z., Schild, S., Bishop, A., et al. (2008). A new Vibrio cholerae sRNA modulates colonization and affects release of outer membrane vesicles. Mol. Microbiol. 70, 100-111. doi: 10.1111/j.1365-2958.2008. 06392.x

Wang, Y., Ke, Y., Xu, J., Wang, L., Wang, T., Liang, H., et al. (2015). Identification of a novel small non-coding RNA modulating the intracellular survival of Brucella melitensis. Front. Microbiol. 6:164. doi: 10.3389/fmicb.2015.00164

Waters, L. S., and Storz, G. (2009). Regulatory RNAs in bacteria. Cell 136, 615-628. doi: 10.1016/j.cell.2009.01.043

Wright, P. R., Georg, J., Mann, M., Sorescu, D. A., Richter, A. S., Lott, S., et al. (2014). CopraRNA and IntaRNA: predicting small RNA targets, networks and interaction domains. Nucleic Acids Res. 42, W119-W123. doi: 10.1093/nar/ gku359

Zhang, X., Ren, J., Li, N., Liu, W., and Wu, Q. (2009). Disruption of the BMEI0066 gene attenuates the virulence of Brucella melitensis and decreases its stress tolerance. Int. J. Biol. Sci. 5, 570-577. doi: 10.7150/ijbs. 5.570

Zhong, Z., Xu, X., Li, X., Liu, S., Lei, S., Yang, M., et al. (2016). Large-scale identification of small noncoding RNA with strand-specific deep sequencing and characterization of a novel virulence-related sRNA in Brucella melitensis. Sci. Rep. 6:25123. doi: 10.1038/srep25123

Conflict of Interest Statement: The authors declare that the research was conducted in the absence of any commercial or financial relationships that could be construed as a potential conflict of interest.

Copyright (c) 2018 Dong, Peng, Liu, Wu, Wang, De, Han, Yuan, Ding, Wang and Wu. This is an open-access article distributed under the terms of the Creative Commons Attribution License (CC BY). The use, distribution or reproduction in other forums is permitted, provided the original author(s) and the copyright owner(s) are credited and that the original publication in this journal is cited, in accordance with accepted academic practice. No use, distribution or reproduction is permitted which does not comply with these terms. 